\title{
Transcriptomic analysis of boron hyperaccumulation mechanisms in Puccinellia distans
}

\author{
Saniye Elvan Öztürk ${ }^{a}$, Mehmet Göktay ${ }^{a}$, Canan Has ${ }^{a}$, Mehmet Babaoğlu ${ }^{b}$, Jens Allmer ${ }^{\text {a }}$, \\ Sami Doğanlar ${ }^{\mathrm{a}}$, Anne Frary ${ }^{\mathrm{a}, *}$ \\ a Department of Molecular Biology and Genetics, Izmir Institute of Technology, Urla, Izmir 35430, Turkey \\ ${ }^{\mathrm{b}}$ Department of Field Crops, Selcuk University, Selçuklu, Konya, 42030, Turkey
}

\section{H I G H L I G H T S}

- Puccinellia distans accumulates extremely high levels of soil boron.

- Hyperaccumulation is accompanied by many transcriptomic changes.

- Changes are seen in the malate pathway and cell wall components.

- Putative boron transporters and aquaporins are up-regulated.

\section{A R T I C L E I N F O}

\section{Article history:}

Received 26 October 2017

Received in revised form

7 February 2018

Accepted 9 February 2018

Available online 12 February 2018

Handling editor: T. Cutright

\section{Keywords:}

Bioremediation

Boron

ESTs

Hyperaccumulation

Puccinellia distans

RNA sequencing

\begin{abstract}
A B S T R A C T
Puccinellia distans, common alkali grass, is found throughout the world and can survive in soils with boron concentrations that are lethal for other plant species. Indeed, $P$. distans accumulates very high levels of this element. Despite these interesting features, very little research has been performed to elucidate the boron tolerance mechanism in this species. In this study, P. distans samples were treated for three weeks with normal $\left(0.5 \mathrm{mg} \mathrm{L}^{-1}\right)$ and elevated $\left(500 \mathrm{mg} \mathrm{L}^{-1}\right)$ boron levels in hydroponic solution. Expressed sequence tags (ESTs) derived from shoot tissue were analyzed by RNA sequencing to identify genes up and down-regulated under boron stress. In this way, 3312 differentially expressed transcripts were detected, $67.7 \%$ of which were up-regulated and $32.3 \%$ of which were down-regulated in borontreated plants. To partially confirm the RNA sequencing results, 32 randomly selected transcripts were analyzed for their expression levels in boron-treated plants. The results agreed with the expected direction of change (up or down-regulation). A total of 1652 transcripts had homologs in A. thaliana and/or $O$. sativa and mapped to 1107 different proteins. Functional annotation of these proteins indicated that the boron tolerance and hyperaccumulation mechanisms of $P$. distans involve many transcriptomic changes including: alterations in the malate pathway, changes in cell wall components that may allow sequestration of excess boron without toxic effects, and increased expression of at least one putative boron transporter and two putative aquaporins. Elucidation of the boron accumulation mechanism is important in developing approaches for bioremediation of boron contaminated soils.
\end{abstract}

() 2018 Elsevier Ltd. All rights reserved.

\section{Introduction}

Boron (B) is a micronutrient with roles in many physiological and biochemical pathways in plants: the metabolism of nucleic acids, carbohydrates, and proteins, cell wall synthesis and structure (Matoh, 2001) and membrane integrity and function (Goldbach,

\footnotetext{
* Corresponding author

E-mail address: annefrary@iyte.edu.tr (A. Frary).
}

1997; Goldbach et al., 2001; Marschner and Marschner, 2012). For example, boron provides stability to the cell wall by crosslinking rhamnogalacturonan-II, a pectic polysaccharide found in the primary wall. The majority of soil boron is found in Turkey ( $72.5 \%$ of world reserves), followed by Russia, USA, and China (BOREN, 2012). Boron levels are affected by rainfall and boron-rich groundwater can cause toxicity in arid and semi-arid regions of the world (Nable et al., 1997; Tanaka and Fujiwara, 2008; Sang et al., 2015). Boron deficiency and toxicity are observed within a very narrow concentration range. Soil concentrations less than $0.5 \mathrm{mg} \mathrm{kg}^{-1}$ are 
associated with deficiency while levels greater than $5.0 \mathrm{mg} \mathrm{kg}^{-1}$ cause toxicity (Ryan and Rashid, 2006). Toxicity alters cell division and reduces cell growth thereby causing decreased crop growth and productivity (Yau et al., 1995; Ozturk et al., 2010). Typical symptoms of boron toxicity are chlorosis and/or necrosis of mature leaf margins and increased cell wall rigidity (Ozturk et al., 2010).

Some plant species tolerate high boron concentrations. One such species, Puccinellia distans (weeping alkali grass) was found to be extremely tolerant of excess boron (Padmanabhan et al., 2012). Under hydroponic conditions, $P$. distans shoots accumulated approximately $6000 \mathrm{mg} \mathrm{kg}^{-1}$ (Bar, 2015). Other work suggested that a related species, Puccinellia frigida, is also highly tolerant to boron with approximately $4000 \mathrm{mg} \mathrm{kg}^{-1}$ accumulation in shoots (Rámila et al., 2015, 2016). Puccinellia species are thus considered important candidates for phytoremediation applications. Compared to chemical, physical and biological boron-removal technologies, phytoremediation and phytoextraction are clean, simple, cost-effective, environmentally friendly methods for removing toxic elements from soil (Padmavathiamma and Li, 2007; Ali et al., 2013; Rámila et al., 2015).

The first boron transport mechanism described in plants was passive transport of uncharged boric acid across the plasma membrane (Marschner and Marschner, 2012). Early studies in bread wheat determined that the $B 01, B 02$, and $B 03$ nuclear genes control boron tolerance (Paull et al., 1992). The BoT1 and BoT2 genes were then identified in barley (Jenkin, 1993) and durum wheat (Jamjod, 1996). Later studies indicated that these genes encode active boron transporter proteins and that boron uptake was hindered by mutations in BOR transporter genes and metabolic inhibitors in various species (Takano et al., 2001; Uraguchi and Fujiwara, 2011). Major intrinsic channel-like transporter proteins (MIPs) also play a role in boron uptake (Dannel et al., 2000; Shinozaki and Yamaguchi-Shinozaki, 2007). Indeed, two aquaporins in $A$. thaliana were shown to have boric acid channel activity (Takano et al., 2006, 2008).

The main goal of this study was to examine the boron hyperaccumulation/tolerance mechanisms of $P$. distans using an RNA sequencing approach. RNA sequencing was employed because transcriptome data provide genome, whole transcriptome and gene level comparisons between treatments as has been shown in other hyperaccumulator plants (Blande et al., 2017; Chen et al., 2017; Yang et al., 2017). P. distans plants were treated with normal $\left(0.5 \mathrm{mg} \mathrm{L}^{-1}\right)$ and elevated $\left(500 \mathrm{mg} \mathrm{L}^{-1}\right)$ boron levels in hydroponic solution and shoot tissues were used for RNA sequencing to identify differentially expressed transcripts. Differentially expressed transcripts were then annotated at the protein level and gene ontology and pathway analyses were performed against $O$. sativa and $A$. thaliana to identify the up and down-regulated genes and proteins that may play roles in $P$. distans boron tolerance and/or hyperaccumulation.

\section{Methods}

\subsection{Plant material and boron treatment}

Seeds of $P$. distans collected from a boron mining site in KirkaEskisehir, Turkey (39 $9^{\circ} 1^{\prime} 23.7156^{\prime \prime}$ and $30^{\circ} 31^{\prime} 33.4812^{\prime \prime}$ ) (Babaoğlu et al., 2004), were germinated in potting soil. The germinated seedlings were grown for 4 weeks in a growth chamber maintained at $25 \pm 2{ }^{\circ} \mathrm{C}, 60 \%$ relative humidity and $16 \mathrm{~h}$ photoperiod (Stiles et al., 2010). Plants were then transferred to half strength Hoagland solution (Hoagland and Arnon, 1950). After a week of growth under hydroponic conditions, plants were separated into two treatment groups with three replicates each: $0.5 \mathrm{mg} \mathrm{L}^{-1}$ boron was applied to the control group and $500 \mathrm{mg} \mathrm{L}^{-1}$ was applied to the stress group (Stiles et al., 2010). The boron concentration in the Hoagland solution was adjusted with boric acid and the solution was changed once every three days for three weeks. After 3 weeks of treatment, plants were removed from the Hoagland solution and rinsed with RNase-free water to remove any contamination. The shoot tissues were then frozen in liquid nitrogen and stored at $-80^{\circ} \mathrm{C}$.

\subsection{RNA isolation}

Total RNA was isolated from the control and boron stressed shoot samples using an RNeasy Plant Mini Kit (Qiagen, Maryland, USA). The quality and quantity of isolated shoot RNA from control and stress samples were measured using a Nanodrop ND-100 device (Nanodrop Technologies, Wilmington, DE, USA).

\subsection{RNA sequencing and analysis}

Total RNA samples were processed using a TruSeqTM RNA Sample Preparation Kit (Illumina, Tokyo, Japan) for cDNA library construction and subsequent EST identification. ESTs were sequenced for control and stress libraries using an Illumina HighSeq 2000 platform (Takara, Tokyo, Japan) to generate $125 \mathrm{bp}$ paired end (PE) reads. RNA sequencing was performed by GATC Biotech (Constance, Germany). Raw data consisted of 45 million reads for each sample with read length fixed at 125 nucleotides.

The Cutadapt2 (version 1.9.1) program (Martin, 2011) was employed with default parameters to remove adapter sequences and low quality nucleotides from raw reads. Reference transcriptome construction was performed using the Trinity (version 2.2.0) assembly tool (PMID: 23845962) (Haas et al., 2013). All reads from the control and stress samples were treated the same. The reference transcriptome was used to map the cleaned reads from control and stress datasets individually using the Bowtie2 program (version 2.1.0) (Langmead and Salzberg, 2012). Mapping information for each dataset was saved in a binary alignment information (bam) file. The bam files were used as inputs for differential gene expression analysis performed with the Cufflinks (version 2.2.1) pipeline (Cufflinks, Cuffmerge, and Cuffdiff) (Trapnell et al., 2012). Cuffdiff selects differentially expressed transcripts based on a $t$-test comparing the normalized counts for each treatment and generates a p-value for each comparison. Results were analyzed and visualized using the cummeRbund (version 2.15) R statistics package (Goff et al., 2013). Differentially expressed gene sequences were selected based on q-value (q-value threshold $<0.05$, where q-value is the false discovery rate adjusted p-value of the test statistic). Differentially expressed candidates were then annotated using Blast2GO (version 4.0.7) (Conesa et al., 2005) against a custom protein database which included Oryza sativa and Arabidopsis thaliana proteins in UniProt Knowledgebase (release date: November 2016). Gene ontology analysis was carried out with QuickGO (Binns et al., 2009). Protein functional classification based on gene ontology was performed by PANTHER Protein Classification System (Accession date: November 2016) (Mi et al., 2017). Annotated transcripts were searched against the KEGG (Kyoto Encyclopedia of Genes and Genomes) database (Tanabe and Kanehisa, 2012) to reveal the pathways in which up and down-regulated transcripts have roles.

\subsection{Evolutionary conservation}

Phylogenetic analyses of boron transporters and other ion transporters of multiple species were performed to determine their sequence similarity and evolutionary conservation. This analysis included all of the fourteen transporters found to be up or 
down-regulated by boron stress in this work: O. sativa boron NIP transporter (UniProtID: Q949A7), A. thaliana TIP transporter (UniProtID: O82598), A. thaliana BOR6 transporter (UniProtID: Q3E954), A. thaliana sugar transporters (UniProtIDs: Q4F7G0, O04249), O. sativa sulfate transporter (UniProtID: Q8S317), O. sativa anion transporter (UniProtID: Q652N5), A. thaliana inorganic phosphate transporter (UniProtID: Q38954), and A. thaliana ABC transporters (UniProtIDs: Q9C9W0, Q8LPJ4, Q7GB25, Q9FWX7, Q9FJH6, Q9M1H3). In addition, 11 boron transporters described in the literature were used in the analysis including those from O. sativa (UniProtIDs: Q1ZYR7, Q7X9F3), A. thaliana (UniprotID: Q8VYR7, Q9XI23, Q93Z13, Q9M1P7, Q9SUU1, Q9SSG5), Hordeum vulgare (UniProtID: M0Z9M9), Brassica napus (UniProtID: D5LGA1, D5LG97). Two A. thaliana NIP transporters (UniProtID: Q9SV84, Q9SAI4) were also included. Sequences for these proteins were retrieved from the UniProtKB database. A. thaliana nuclear transport factor 2 (NTF2) protein (UniprotID: F4J8X6) was included as outgroup.

Multiple sequence alignment was conducted with the default settings of ClustalW (Larkin et al., 2007). All positions with continuous alignment gaps were eliminated using the MEGA 7.0 suite (Tamura et al., 2013). Phylogenetic tree construction was performed using the maximum likelihood method based on the Le Gascuel substitution model (Le and Gascuel, 2008). The Gamma distribution was used to model evolutionary rate differences among sites (with default parameters except for $G$ which was set to 13,3340 ). Bootstrap values were inferred from 1000 replicates. In order to compute phylogenetic distances, the boron and other transporters were separated into two groups. Within and between groups distances were calculated by computing the number of amino acid differences per site, averaged over all sequence pairs.

\subsection{Quantitative real-time $P C R$}

The expression of 30 genes that responded to boron stress in P. distans shoots was confirmed by qRT-PCR (quantitative real-time polymerase chain reaction) analysis using three technical replicates from one of the three biological replicates used for RNA-seq analysis. The extracted total RNA was treated with DNase I (Takara, Shiga, Japan). GoTaq ${ }^{\circledR}$ 2-Step RT-qPCR kit (PROMEGA) was used for CDNA synthesis and the resulting CDNAs were amplified in the LightCycler ${ }^{\circledR} 480$ system (Roche, Basel, Switzerland) using transcript-specific primers (Table S7). The 30 transcripts were selected randomly from the top 50 up and down-regulated transcripts. In addition, primers were used for one known $A B C$ transporter and one known boron transporter (Padmanabhan et al., 2012). Differences in gene expression were analyzed by one-way ANOVA followed by Dunnett's $t$-test for comparisons between groups using GraphPad Prism 6.0 software (Graphpad Software Inc, San Diego, CA USA). A value of $\mathrm{P}<0.05$ was considered statistically significant as compared with the control (Schmidt et al., 2017; Tian et al., 2017). Actin was used as internal control and error bars indicate standard deviation of three technical repeats. ${ }^{* * *}$ indicates significant difference at $\mathrm{p} \leq 0.001,{ }^{* *}$ indicates significant difference at $\mathrm{p} \leq 0.01$ and ${ }^{*}$ indicates significant difference at $\mathrm{p} \leq 0.05$ with respect to control.

\section{Results}

Phytoremediation and phytoextraction are clean, simple, costeffective, environmentally friendly methods for removing toxic elements from soil (Rámila et al., 2015). These methods rely on plants similar to $P$. distans, an extreme hyperaccumulator of boron. Elucidation of the mechanism(s) by which $P$. distans accumulates and tolerates normally toxic levels of boron may allow development of a faster growing, higher biomass boron hyperaccumulator. This study provides the first broad examination of the transcriptomic response of $P$. distans to boron hyperaccumulation.

\subsection{Analysis of the P. distans transcriptome with RNA-seq}

To investigate the transcriptomic response of $P$. distans to boron stress, four cDNA libraries were generated from two replicate mRNA samples from boron-treated and control shoot samples. These libraries were sequenced using Illumina deep-sequencing HiSeq $^{\mathrm{TM}}$ 2000. Raw reads of control and boron-treated samples were cleaned of adapter sequences using the Cutadapt2 tool resulting in $88,663,592$ (98.0\%) and $82,494,636$ (92.4\%) reads for control and stress samples, respectively.

The cleaned reads from the two samples were treated as input for the Trinity assembler. All reads were mapped to the constructed reference transcriptome individually using the Bowtie2 program. Mapping ratios for control and stress samples were $97.8 \%$ and $97.3 \%$ of cleaned reads, respectively. Based on transcripts that mapped to the reference transcriptome, 284,371,845 bases were assembled from 179,792,482 reads (control + stress) (Table 1$)$. Median and average contig lengths were 317 and $\sim 497 \mathrm{nt}$, respectively (Table 1). Additionally, the N50 value indicated that at least half the assembled bases were found in contigs that were at least $609 \mathrm{nt}$ long (Table 1).

\subsection{Differential gene expression between control and stress conditions}

Differentially expressed transcripts were detected using the RNA-Seq alignments and Cufflinks processing. Cuffdiff tracked the mapped reads and determined the fragments per kb per million mapped reads (FPKM) for each transcript in all samples. These FPKM values were used to detect statistically significant up and down-regulated transcripts and most differential transcripts were found to be present or absent under control or stress conditions (Fig. S1). Of 3312 differentially expressed transcripts, 2242 (67.7\%) were up-regulated while 1070 (32.3\%) were down-regulated (Table S1).

\subsection{Annotation of differentially expressed transcripts}

The number of $P$. distans proteins and annotations available in public data repositories is limited. Therefore, $P$. distans annotation was conducted using the protein databases of two model organisms: Arabidopsis thaliana and Oryza sativa. Both of these have extensive protein sequence resources available in the UniProt knowledgebase repository. In addition, $O$. sativa and $P$. distans belong to the same family, Gramineae, indicating a close genetic relationship (Wang et al., 2007). A total of 3312 transcripts were found to be differentially expressed. Of these, 1660 transcripts had no homologs in $A$. thaliana or $O$. sativa. Thus, these transcripts may be specific to $P$. distans and some may play roles in boron hyperaccumulation, a mechanism which is not present in $A$. thaliana and

Table 1

Summary of transcripts/genes statistics of $P$. distans.

\begin{tabular}{ll}
\hline Parameter & \\
\hline Number of Reads (Control + Stress) & $179,792,482$ \\
Average Raw Read Length (Control + Stress) & $125 \mathrm{nt}$ \\
Average Contig Length & $496.73 \mathrm{nt}$ \\
Median Contig Length & $317 \mathrm{nt}$ \\
N50 & $609 \mathrm{nt}$ \\
Total Assembly Size & $284,371,845 \mathrm{nt}$ \\
\hline
\end{tabular}


O. sativa. The remaining 1652 (49.9\%) transcripts mapped to 1107 proteins. Multiple transcripts (a total of 335 transcripts) matching 36 proteins were labelled as conflicting due to matching both up and down-regulated transcripts according to their FPKM values. In order to resolve this, BLAST results were inspected manually. In this way, 36 transcripts with the highest sequence identity and best hit score were kept and the remaining transcripts were discarded as non-annotated. Among the annotated transcripts, 28 matched 21 different transporter proteins including known B transporters and aquaporins. (Table S2). The annotations for the most differentially expressed transcripts (based on stress and control FPKM values) are listed in Table 2. Transcripts that did not return any match against rice and arabidopsis proteins were annotated against the green plant database (Viridiplantae) from UniProt Knowledgebase (http://www.uniprot.org/taxonomy/33090, release date: November 2016). However, no additional candidate proteins were found at a significance level of match based on best alignment length and E-value (E-value < 1e-5) (data not shown).

Functional annotation was performed using Blast2GO and QuickGO based on gene ontology (GO). Cellular component, molecular function, and biological process were analyzed as GO terms for the 1107 annotated proteins. According to the ontology analysis,
$906(81.8 \%)$ proteins were annotated to cellular components. 885 $(80.0 \%)$ and 848 (76.6\%) proteins were annotated with their molecular functions and biological processes, respectively.

\subsection{Cellular components}

Cellular component analysis connected 906 proteins $(81.8 \%)$ to at least one cellular component-associated term (Table S3). Because excess boron is trafficked from the plasma membrane to other organelles such as the vacuole and cell wall to avoid boron toxicity (Hanaoka et al., 2014), only these cellular component-associated GO terms are discussed here. A total of $14.8 \%$ of proteins were associated with the integral component of the membrane, plasma membrane, vacuolar membrane, mitochondrial inner membrane and endoplasmic reticulum membrane. In addition, $1.4 \%$ of proteins were related to the cell wall, $0.8 \%$ were localized in the apoplast and $1.5 \%$ were in the vacuole.

\subsection{Biological processes}

Biological process annotations fell into seven main categories: response to stimulus, cellular process, metabolic process,

Table 2

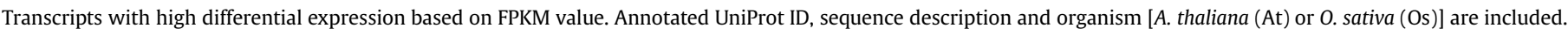

\begin{tabular}{|c|c|c|c|}
\hline UniProtID/Annotation & Organism & Transcript Name & Difference $^{\mathrm{a}}$ \\
\hline F4I600_ARATH/V-ATPase-related & At & TRINITY_DN213899_c1_g5_i1:0-169 & $20,561.12$ \\
\hline H32_ARATH/Histone & At & TRINITY_DN99923_c0_g1_i1:0-171 & 845.90 \\
\hline RRAA2_ARATH/4-hydroxy-4-methyl-2-oxoglutarate aldolase 2 & At & TRINITY_DN220021_c1_g4_i1:0-584 & 426.28 \\
\hline PSBR_ARATH/Photosystem II 10 kDa chloroplastic & At & TRINITY_DN192927_c1_g1_i1:132-532 & 329.61 \\
\hline RD22_ARATH/BURP domain RD22 & At & TRINITY_DN131860_c0_g2_i1:0-222 & 315.95 \\
\hline RRAA3_ARATH/4-hydroxy-4-methyl-2-oxoglutarate aldolase 3 & At & TRINITY_DN220021_c1_g4_i2:0-391 & 299.33 \\
\hline RRAA3_ARATH/4-hydroxy-4-methyl-2-oxoglutarate aldolase 3 & At & TRINITY_DN214561_c1_g18_i1:0-365 & 253.61 \\
\hline H2B10_ARATH/Histone & At & TRINITY_DN199272_c0_g3_i1:2-246 & 168.98 \\
\hline H2B1_ARATH/Histone & At & TRINITY_DN150574_c0_g1_i1:0-201 & 130.08 \\
\hline A0A160DR54_ORYSA/Dirigent (Fragment) & Os & TRINITY_DN215985_c1_g3_i1:4-592 & 126.38 \\
\hline RL40B_ARATH/Ubiquitin-60S ribosomal L40-2 & At & TRINITY_DN67858_c0_g1_i1:0-219 & 85.64 \\
\hline H2A1_ARATH/Probable histone & At & TRINITY_DN28983_c0_g1_i1:38-250 & 80.83 \\
\hline FRO7_ARATH/Ferric reduction oxidase chloroplastic & At & TRINITY_DN224067_c1_g1_i1:0-1399 & 79.87 \\
\hline H32_ARATH/Histone & At & TRINITY_DN271392_c0_g1_i1:0-479 & 66.13 \\
\hline H2B3_ARATH/Histone & At & TRINITY_DN199272_c0_g1_i1:0-245 & 65.63 \\
\hline H2A1_ARATH/Probable histone & At & TRINITY_DN113560_c0_g1_i1:0-238 & 63.07 \\
\hline XTH22_ARATH/Xyloglucan endotransglucosylase hydrolase 22 & At & TRINITY_DN221642_c3_g12_i1:2-232 & 57.72 \\
\hline H32_ARATH/Histone & At & TRINITY_DN48466_c0_g1_i1:30-384 & 51.59 \\
\hline Q01KB9_ORYSA/Autophagy-related & Os & TRINITY_DN202627_c0_g7_i1:92-353 & 45.84 \\
\hline ACT3_ARATH/Actin-3 & At & TRINITY_DN272586_c0_g1_i1:1-261 & 39.90 \\
\hline SYHM_ARATH/Histidine-tRNA chloroplastic mitochondrial & At & TRINITY_DN179460_c0_g1_i1:0-227 & 39.49 \\
\hline COX3_ARATH/Cytochrome $c$ oxidase subunit 3 & At & TRINITY_DN264216_c0_g1_i1:1-257 & 38.02 \\
\hline H4_ARATH/Histone H4 & At & TRINITY_DN8209_c0_g1_i1:0-419 & 31.53 \\
\hline Q949A7_ORYSA/aquaporin (Fragment) & Os & TRINITY_DN210823_c0_g3_i1:4-269 & 30.12 \\
\hline H2A1_ARATH/Probable histone & At & TRINITY_DN202443_c4_g5_i3:0-460 & 29.44 \\
\hline FRS5_ARATH/FAR1-RELATED SEQUENCE 5 & At & TRINITY_DN114402_c0_g1_i1:9-274 & 29.40 \\
\hline H2A1_ARATH/Probable histone & At & TRINITY_DN202443_c4_g4_i2:0-314 & 25.88 \\
\hline H2A1_ARATH/Probable histone & At & TRINITY_DN202443_c4_g4_i3:0-340 & 25.53 \\
\hline Q9LK47_ARATH/AT3g23700 MYM9_3 & At & TRINITY_DN219995_c1_g16_i1:0-256 & 25.08 \\
\hline A0A0KOWRF9_ORYSA/NBS-LRR type R & Os & TRINITY_DN226626_c0_g8_i1:3-253 & 24.76 \\
\hline H2A1_ARATH/Probable histone & At & TRINITY_DN202443_c4_g1_i1:0-255 & 24.25 \\
\hline NEN3_ARATH/NEN3 & At & TRINITY_DN226592_c4_g15_i1:8-257 & 21.50 \\
\hline RL341_ARATH/60S ribosomal L34-1 & At & TRINITY_DN295721_c0_g1_i1:0-414 & 20.41 \\
\hline F4HS76_ARATH/Glycerol kinase & At & TRINITY_DN140573_c0_g2_i1:0-250 & 20.29 \\
\hline RL142_ARATH/60S ribosomal L14-2 & At & TRINITY_DN179003_c1_g2_i1:7-283 & 20.12 \\
\hline RL74_ARATH/60S ribosomal L7-4 & At & TRINITY_DN294520_c0_g1_i1:0-828 & 20.02 \\
\hline F4IJE1_ARATH/Phox domain-containing & At & TRINITY_DN278982_c0_g1_i1:1-268 & 19.68 \\
\hline COX2_ARATH/Cytochrome $c$ oxidase subunit 2 & At & TRINITY_DN284184_c0_g1_i1:0-703 & 19.48 \\
\hline Y1457_ARATH/Acyltransferase chloroplastic & At & TRINITY_DN222292_c3_g2_i1:0-281 & 18.69 \\
\hline Q9LTW8_ARATH/CTP synthase & At & TRINITY_DN1712_c0_g1_i1:0-285 & 18.65 \\
\hline A0A0U2JFK9_ORYSA/NBS-LRR-like resistance & Os & TRINITY_DN213110_c2_g2_i4:22-323 & 16.98 \\
\hline UBQ3_ARATH/Polyubiquitin 3 & At & TRINITY_DN186267_c2_g1_i1:18-638 & 16.88 \\
\hline ACT11_ARATH/Actin-11 & At & TRINITY_DN204305_c3_g6_i2:0-375 & 16.85 \\
\hline A8MRY7_ARATH/WD-40 PCN & At & TRINITY_DN224769_c1_g7_i1:4-287 & 15.55 \\
\hline Q9STF8_ARATH/PR-6ase inhibitor family & At & TRINITY_DN127914_c0_g2_i1:0-296 & 15.37 \\
\hline
\end{tabular}

\footnotetext{
${ }^{a}$ Difference: FPKM (Fragments per Kilobase of Exon per Million Fragments Mapped) difference between control and stress conditions.
} 
localization, reproductive process, developmental process and signaling (Table S4). Proteins related to plant responses to hormones and stress conditions such as salt, various ions, cold, heat, oxidative stress, and water deprivation were identified. Our findings indicated that not only translation and transcription but also protein folding and turnover, cell wall organization and biogenesis, and ion dependent redox homeostasis were regulated under excess boron. Both anabolic processes such as the malate-fumarate pathway and catabolic processes such as protein and lignin pathways were altered under stress conditions. Proteins that play roles in signal transduction, in particular plant hormone-mediated and sugar-mediated signaling pathways were also differentially regulated.

\subsection{Molecular functions}

Molecular function annotation of transcripts resulted in three main categories: transporter activity, catalytic activity and binding (Table 3). The most abundant molecular function categories were binding activity (39\%) and catalytic activity (67\%). Binding activity included copper, zinc, calcium, manganese, cobalt and ferritin binding indicating that excess boron alters signal transduction and vesicle trafficking in the cell. Catalytic activities of enzymes such as serine/threonine kinase, GTPase, ATPase, $\mathrm{H}^{+}$transporting ATP synthase, and oxidoreductase were regulated under boron stress. Differentially expressed transporters included proton, glucose, malate, inorganic phosphate, L-ascorbic acid, L-glutamate, auxin, and ion transporters. Transporters for minerals including calcium, sulfate, and manganese were also identified in this category. Moreover, three previously identified boron transporters were found: A. thaliana BOR6 putative boron transporter protein (Transcript Name: TRINITY_DN188130_c0_g1_i1:0-1084), A. thaliana TIP1-3 protein (Transcript Name: TRINITY_DN222891_c3_g1_i1:0216), and 0 . sativa NIP protein (Transcript Name: TRINITY_DN210823_c0_g3_i1:4-269).

Annotated transcripts were uploaded to the KEGG system for metabolic pathway analysis (Kanehisa, 2002). A total of 756 proteins were found to have interactions with pathways in the KEGG database (Table S5). In this database, 327 (43.2\%) proteins fell into the general metabolism category. Of these, 66 were involved in carbon metabolism, 64 in energy metabolism, 50 in amino acid metabolism, 31 in nucleic acid metabolism, 24 in lipid metabolism and 7 in secondary metabolism. The remaining 34 proteins were placed in other metabolism-related subcategories. A total of 387 proteins $(51.2 \%)$ were in the genetic information process category. Of these, the majority (184) fell into the translation subcategory. The protein folding category was the second most populous group in genetic information processes with 98 proteins. Environmental information processes were also observed for 152 proteins (20.1\%) with 148 proteins matching signal transduction and four matching membrane transport. Finally, 152 proteins (20.1\%) were associated with cellular processes. In this category, two subgroups resulted in high protein matches: 86 proteins in the cellular growth and death subcategory and 42 proteins in the transport and catabolism subcategory.

\subsection{Evolutionary relationships among transporters}

$O$. sativa and $A$. thaliana are not boron hyperaccumulating plants; therefore, boron transporters in $P$. distans may have been annotated to other ion transporters in these species. Phylogenetic analysis was used to determine the degree of evolutionary similarity between known boron transporters (from $O$. sativa, A. thaliana, B. napus, and $H$. vulgare) and the annotated $A$. thaliana and $O$. sativa boron (BOR6), aquaporin (NIP and TIP), sugar, sulfate, anion, inorganic phosphate, and $\mathrm{ABC}$ transporters from our dataset (Fig. 1). A total of 28 amino acid sequences were compared over 160 positions. The samples included an $A$. thaliana nuclear transport factor protein as outgroup. The phylogenetic analysis indicated that the identified sugar, sulfate, anion, inorganic phosphate and $A B C$ transporter proteins were distinct from boron transporters which were also distinct from aquaporins. The mean p-distance was $30 \%$ between boron transporters and $60 \%$ between aquaporins. Wakuta et al. (2015) examined the evolutionary relationships among boron transporters in terrestrial plants. According to this work, boron transporters fell into two major and one minor clade that may reflect their physiological differences. Clade I contained transporters such as BOR1 that are important under limited boron conditions and are responsible for directional export of boron in the plant. Clade II contained transporters such as BOR6 that are important under high boron conditions and are responsible for boron exclusion. Our phylogenetic analysis agreed with that of Wakuta et al. in separating the BOR1, 2 and 3 homologs from BOR6 and 7.

\subsection{Real-time PCR results of annotated transcripts}

A total of 32 transcripts were selected for verification of their expression levels using quantitative real time-PCR (qRT-PCR). Transcripts were chosen randomly based on their differential expression between control and stress samples. Up and downregulated transcripts are shown in Fig. 2. The most significant five up-regulated transcripts were annotated to: an aquaporin of O. sativa (DN210823); a photosystem II protein in Arabidopsis (DN192927); a 4-hydroxy-4-methyl-2-oxoglutarate aldolase in A. thaliana (DN220021); a ferric reduction oxidase in $A$. thaliana (DN224067); and FRIGIDA, which is required for FLC (Flowering Locus C) activity in A. thaliana (DN111068) (Fig. 2A). Significant down-regulation was observed for four transcripts: three of which were annotated to have oxidoreductase activities in 0 . sativa (DN212455, DN205322, DN216505) and one that was annotated to aquaporin TIP1-3 in A. thaliana (DN222891) (Fig. 2B; Table S6).

\section{Discussion}

Plants activate a wide range of functional and regulatory genes to avoid or tolerate disruptive situations that accompany environmental stresses such as drought, cold, salinity and metalloid accumulation (Shinozaki et al., 2003; Shinozaki and YamaguchiShinozaki, 2007). Many of these genes play roles in basic plant metabolism and are not specific to the stress response. Therefore, we will only focus on candidate genes for stress-related and cell wall proteins, transporters and plant hormones that are differentially regulated under excess boron.

\subsection{Stress and cell wall-related genes}

According to KEGG analysis, certain stress-related molecules were highly active under excess boron as compared to normal boron levels. For example, the $A$. thaliana homolog of UDPglycosyltransferase (UniProtID: U88A1_ARATH; Transcript Name: TRINITY_DN223147_c5_g2_i1:16-443), which plays a role in flavonoid biosynthesis, was highly up-regulated (Table S6). Flavonoids are well-known antioxidants and are important in detoxifying the excess free radicals that plants produce under stress.

The ubiquitin/26S proteasome pathway is important for protein degradation and controls protein level and activity. In general, 26S proteasome activity is induced under stress conditions and possibly this mechanism has a role in plant adaptation to extreme B conditions (Tombuloglu et al., 2017). For example, its activity was 
Table 3

Molecular function main and subcategories which were most related to boron hyperaccumulation in P. distans.

\begin{tabular}{|c|c|}
\hline Molecular Function GOTerm & Percentage of proteins \\
\hline Binding & $39.00 \%$ \\
\hline ATP binding & $9.90 \%$ \\
\hline Metal ion binding & $4.60 \%$ \\
\hline RNA binding & $3.80 \%$ \\
\hline DNA binding & $3.70 \%$ \\
\hline Zinc ion binding & $3.20 \%$ \\
\hline GTP binding & $1.60 \%$ \\
\hline Translation initiation factor activity & $1.60 \%$ \\
\hline Nucleic acid binding & $1.50 \%$ \\
\hline Copper ion binding & $1.40 \%$ \\
\hline Transcription factor activity, sequence-specific DNA binding & $1.10 \%$ \\
\hline ADP binding & $1.00 \%$ \\
\hline Heme binding & $1.00 \%$ \\
\hline Calcium ion binding & $0.60 \%$ \\
\hline Iron ion binding & $0.50 \%$ \\
\hline Magnesium ion binding & $0.30 \%$ \\
\hline HSP90 protein binding & $0.20 \%$ \\
\hline 4 iron, 4 sulfur cluster binding & $0.20 \%$ \\
\hline Cobalt ion binding & $0.10 \%$ \\
\hline Manganese ion binding & $0.10 \%$ \\
\hline 2 iron, 2 sulfur cluster binding & $0.10 \%$ \\
\hline Four-way junction DNA binding & $0.10 \%$ \\
\hline Calmodulin binding & $0.10 \%$ \\
\hline Quinone binding & $0.10 \%$ \\
\hline Iron-sulfur cluster binding & $0.10 \%$ \\
\hline Catalytic activity & $67.00 \%$ \\
\hline Structural constituent of ribosome & $4.90 \%$ \\
\hline Protein serine/threonine kinase activity & $1.30 \%$ \\
\hline Protein kinase activity & $1.00 \%$ \\
\hline GTPase activity & $0.90 \%$ \\
\hline ATP-dependent RNA helicase activity & $0.80 \%$ \\
\hline ATPase activity & $0.80 \%$ \\
\hline NADH dehydrogenase (ubiquinone) activity & $0.60 \%$ \\
\hline Proton-transporting ATP synthase activity, rotational mechanism & $0.60 \%$ \\
\hline Structural molecule activity & $0.60 \%$ \\
\hline Oxidoreductase activity & $0.60 \%$ \\
\hline Kinase activity & $0.50 \%$ \\
\hline Signal transducer activity & $0.30 \%$ \\
\hline Peroxidase activity & $0.30 \%$ \\
\hline Metalloendopeptidase activity & $0.30 \%$ \\
\hline Cytochrome-c oxidase activity & $0.30 \%$ \\
\hline Fumarate hydratase activity & $0.20 \%$ \\
\hline Metalloaminopeptidase activity & $0.10 \%$ \\
\hline Calcium-dependent cysteine-type endopeptidase activity & $0.10 \%$ \\
\hline Glutathione transferase activity & $0.10 \%$ \\
\hline Peroxiredoxin activity & $0.10 \%$ \\
\hline Oxidoreductase activity, acting on the $\mathrm{CH}-\mathrm{CH}$ group of donors, with a flavin as acceptor & $0.10 \%$ \\
\hline Glutathione peroxidase activity & $0.10 \%$ \\
\hline Calcium-transporting ATPase activity & $0.10 \%$ \\
\hline Electron transporter, transferring electrons from $\mathrm{COQH} 2$-cytochrome $\mathrm{c}$ reductase complex and cytochrome $c$ oxidase complex activity & $0.10 \%$ \\
\hline L-lactate dehydrogenase activity & $0.10 \%$ \\
\hline L-malate dehydrogenase activity & $0.10 \%$ \\
\hline Metallopeptidase activity & $0.10 \%$ \\
\hline Ferric-chelate reductase activity & $0.10 \%$ \\
\hline Substrate-specific transmembrane transporter activity & $0.10 \%$ \\
\hline Oxaloacetate decarboxylase activity & $0.10 \%$ \\
\hline Manganese-transporting ATPase activity & $0.10 \%$ \\
\hline Oxidoreductase activity, acting on paired donors, with incorporation or reduction of molecular oxygen & $0.10 \%$ \\
\hline Malate dehydrogenase (decarboxylating) (NADP+) activity & $0.10 \%$ \\
\hline Oxidoreductase activity, acting on the $\mathrm{CH}-\mathrm{CH}$ group of donors & $0.10 \%$ \\
\hline Oxalate decarboxylase activity & $0.10 \%$ \\
\hline $\begin{array}{l}\text { Oxidoreductase activity, acting on paired donors, with oxidation of a pair of donors resulting in the reduction of } \\
\text { molecular oxygen to two molecules of water }\end{array}$ & $0.10 \%$ \\
\hline L-ascorbate peroxidase activity & $0.10 \%$ \\
\hline Cytochrome-c peroxidase activity & $0.10 \%$ \\
\hline Glutamate decarboxylase activity & $0.10 \%$ \\
\hline Myo-inositol & $0.10 \%$ \\
\hline Oxidoreductase activity, acting on the $\mathrm{CH}-\mathrm{CH}$ group of donors, NAD or NADP as acceptor & $0.10 \%$ \\
\hline Nuclear export signal receptor activity & $0.10 \%$ \\
\hline Oxidoreductase activity, acting on $\mathrm{NAD}(\mathrm{P}) \mathrm{H}$, heme protein as acceptor & $0.10 \%$ \\
\hline Aspartate-tRNA ligase activity & $0.10 \%$ \\
\hline Malate dehydrogenase (decarboxylating) (NAD+) activity & $0.10 \%$ \\
\hline
\end{tabular}


Table 3 (continued)

\begin{tabular}{|c|c|}
\hline Molecular Function GOTerm & Percentage of proteins \\
\hline Oxidoreductase activity, oxidizing metal ions & $0.10 \%$ \\
\hline Protein tyrosine/serine/threonine phosphatase activity & $0.10 \%$ \\
\hline Transporter activity & $9.00 \%$ \\
\hline Transporter activity & $0.40 \%$ \\
\hline Glucose transmembrane transporter activity & $0.20 \%$ \\
\hline Sugar:proton symporter activity & $0.20 \%$ \\
\hline Inorganic phosphate transmembrane transporter activity & $0.20 \%$ \\
\hline Malate transmembrane transporter activity & $0.10 \%$ \\
\hline L-ascorbic acid transporter activity & $0.10 \%$ \\
\hline Secondary active sulfate transmembrane transporter activity & $0.10 \%$ \\
\hline Antiporter activity & $0.10 \%$ \\
\hline Voltage-gated ion channel activity & $0.10 \%$ \\
\hline Anion:anion antiporter activity & $0.10 \%$ \\
\hline Auxin efflux transmembrane transporter activity & $0.10 \%$ \\
\hline Outward rectifier potassium channel activity & $0.10 \%$ \\
\hline Ion transmembrane transporter activity & $0.10 \%$ \\
\hline Ion channel activity & $0.10 \%$ \\
\hline Channel regulator activity & $0.10 \%$ \\
\hline Inorganic anion exchanger activity & $0.10 \%$ \\
\hline Anion transmembrane transporter activity & $0.10 \%$ \\
\hline Inward rectifier potassium channel activity & $0.10 \%$ \\
\hline L-glutamate transmembrane transporter activity & $0.10 \%$ \\
\hline Zinc ion transmembrane transporter activity & $0.10 \%$ \\
\hline Porin activity & $0.10 \%$ \\
\hline
\end{tabular}

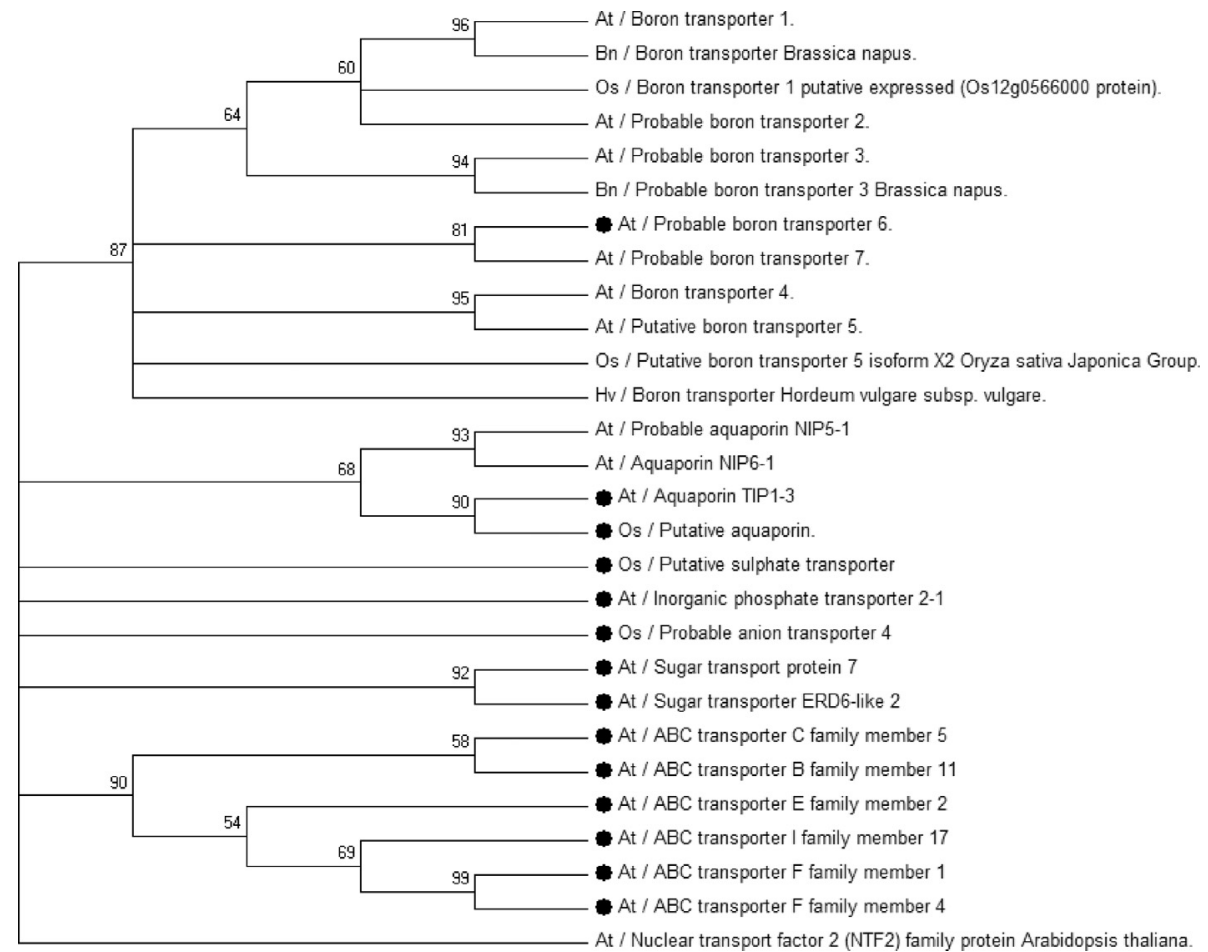

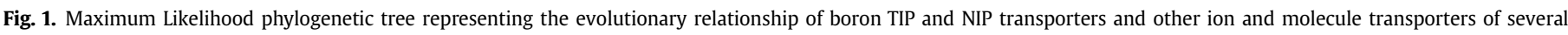

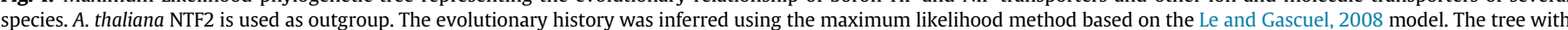

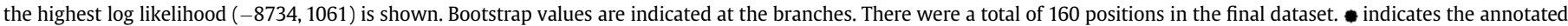
transcripts from this study.

calculated as 11.46 fold higher under boron stress in Gypsophila perfoliata, a boron hyperaccumulator plant (Unver et al., 2008). Similarly, 26S proteasome and its subunits were upregulated under excess boron in the present study. A possible explanation is that the 26S proteasome machinery is important to degrade proteins damaged as a result of toxicity.

The malate pathway was also affected by high boron stress. Activation of this pathway is correlated with abiotic stress (Kumar et al., 2000). Four malate-related A. thaliana homolog proteins
(UniProtIDs: B3H477_ARATH, FUM1_ARATH, FUM2_ARATH, and MAOP3_ARATH) were down-regulated under excess boron. In contrast, malate dehydrogenase 1 enzyme, which is responsible for converting malate to oxaloacetate, was up-regulated (UniProtID: MDHC1_ARATH) (Table S6). This combined increase in malate dehydrogenase 1 and decreased expression of two fumarate hydratases (UniProtIDs: FUM1-2 and B3H477) under boron stress indicates altered regulation in the glyoxylate pathway at malate (Fig. 3A) which is in line with previous findings (Beevers et al., 

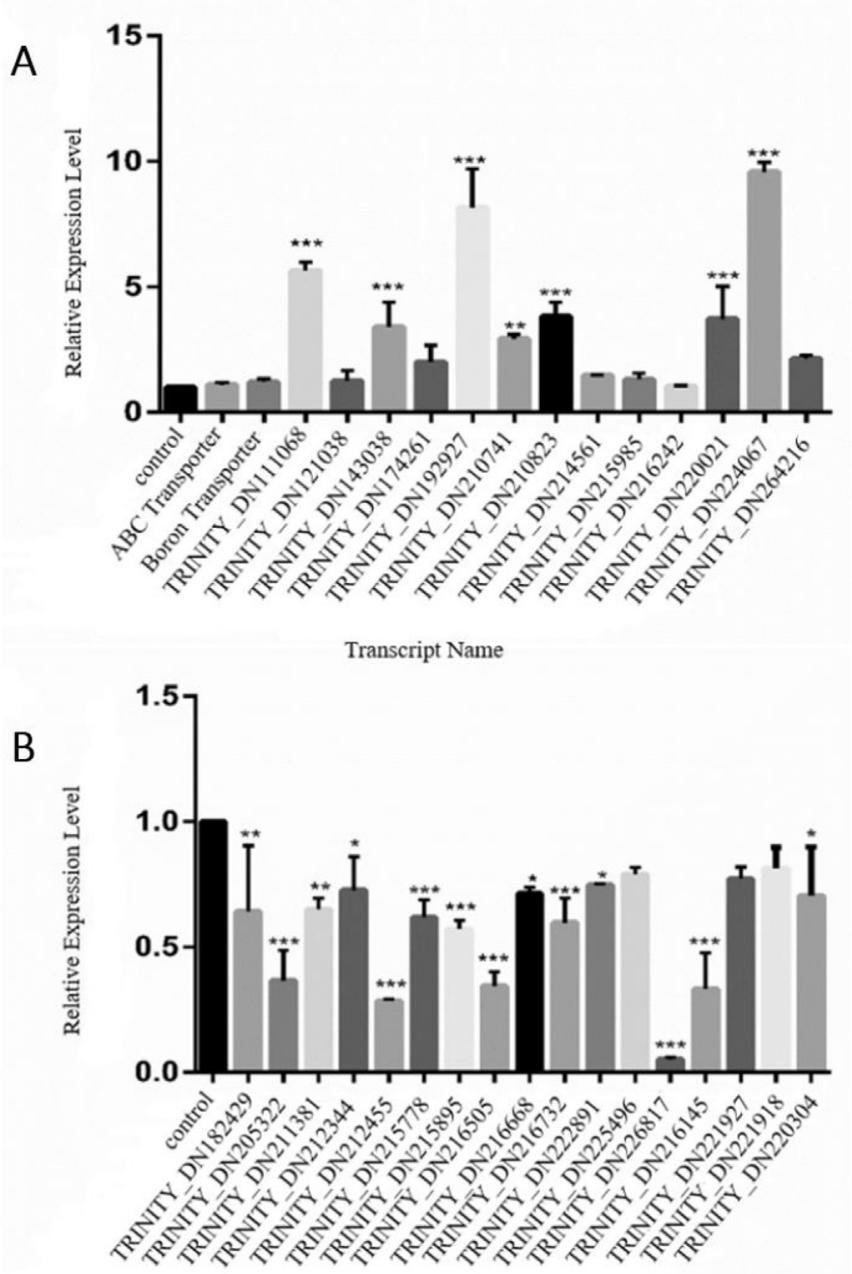

Transcript Name

Fig. 2. Real-time PCR results of $P$. distans transcriptome for 32 selected transcripts. (A) Fifteen of the transcripts were confirmed to be up-regulated; while 17 were confirmed to be down-regulated (B) Actin was used as internal control and error bars indicate standard deviation of three technical repeats. ${ }^{* * *}$ indicates significant difference at $\mathrm{p} \leq 0.001$, ** indicates significant difference at $\mathrm{p} \leq 0.01$ and ${ }^{*}$ indicates significant difference at $\mathrm{p} \leq 0.05$ with respect to control.

2014). In this pathway, isocitrate is converted to malate via the intermediate molecule, glyoxylate. Glyoxylate and pyruvate can also be formed by a low affinity reaction catalyzed by 4-hydroxy-4methyl-2-oxoglutarate aldolase (Fig. 3B) (Maruyama, 1990). In boron-stressed plants, two 4-hydroxy-4-methyl-2-oxoglutarate aldolases (RRAA2_ARATH and RRAA3_ARATH) were significantly up-regulated (FPKMs: 426.28 and 253.61, respectively) (Table S6). High activity of these aldolases can result in increased glyoxylate which is converted to malate. Malate can then be transported out of the cell with ions like $\mathrm{K}^{+}$in order to balance cellular $\mathrm{pH}$, a mechanism that is known to be a response to aluminum tolerance in wheat (Ryan et al., 1995). Thus, these results indicate that malate and glyoxylate pathway proteins have roles in boron hyperaccumulation.

Depending on plant species, sugars (such as sucrose), sugar alcohols (such as mannitol) and amino acids (such as glycine) accumulate to help plants cope under stress conditions (Taji et al., 2002; Bartels and Sunkar, 2005). Sugar alcohols provide protection from stress by scavenging hydroxyl radicals and/or stabilizing macromolecular structure (Seki et al., 2007). Inositol is a sugar alcohol which also has a role in the biogenesis of the uronosyl and pentosyl units of pectin, hemicelluloses, and related structures in plant cell walls (Loewus and Loewus, 1983; Loewusa and Murthy, 2000). An inositol transporter was up-regulated in $P$. distans (UniProtID: INT2_ARATH; Transcript name: TRINITY_DN172179_c0_g2_i1:0$695,2.5518)$ under high boron. An increased level of this transporter could be used in the cell to increase the level of inositol available to scavenge reactive oxygen species and/or chelate boron, thereby protecting the cell. Increased cellular inositol may also be required for the synthesis of cell wall pectin and its precursor to bind to the increased amount of boron in the cell wall. The similarity between the inositol and boron transporters of $A$. thaliana (Tanaka and Fujiwara, 2008) suggests a third possibility: the inositol transporter may also carry boron.

Plant cells are enclosed within cell walls which form physical barriers and provide tolerance to turgor pressure (Baskin, 2005). The cell wall consists of polysaccharides, proteins and phenolic compounds (Levy and Staehelin, 1992). Boron is another important structural component of the cell wall as it crosslinks rhamnogalacturonan-II molecules, thereby providing stability. Expansin proteins are also found in the wall and are responsible for cell enlargement by loosening the wall (Cosgrove, 2005). Expansins directly interact with RD22 proteins to mediate cell enlargement (Wang et al., 2012). RD22 proteins contain a BURP domain, an amino acid sequence which is highly conserved in plant species (Xu et al., 2010). An acronym, BURP comes from: BNM2 (Brassica napus microspore-derived embryos protein) (Treacy et al., 1997), USP (Vicia faba; unidentified seed protein) (Bassüner et al., 1988), RD22 (A. thaliana protein responsive to desiccation) (YamaguchiShinozaki and Shinozaki, 1993), and PG1 $\beta$ (Solanum lycopersicum $\beta$ subunit of polygalacturonase isozyme 1) (Zheng, 1992). Here we detected the upregulation of the A. thaliana homolog of the RD22 protein (Table 3). In addition to its role in cell enlargement, RD22 was reported to be responsible for increased levels of lignin precursor in cell walls under salt stress (Wang et al., 2012). Excess boron has been shown to interact with lignin precursors in Pinus radiata organ cultures (Reid, 2007). Therefore, the up-regulated RD22 homolog in $P$. distans may provide additional lignin precursors, thereby increasing the boron binding capacity of cell walls under stress. Thus, RD22 may be an important component of the hyperaccumulation mechanism in $P$. distans. Similarly, BURP domain activity was also upregulated in rice under boron toxicity (Neto et al., 2017).

\subsection{Transporter genes}

Transporter proteins have been shown to play important roles in coping with stress in the boron hyperaccumulator plants, poplar and Puccinellia (Padmanabhan et al., 2012; Rámila et al., 2016; Yildirim and Uylas, 2016). Indeed, the first identified boron tolerance-related gene was Bot1 which codes for a putative transmembrane B efflux transporter in barley (Reid, 2007). Many studies on boron efflux transporters have shown that the presence of these transporters is controlled by cellular boron concentration. Miwa et al. (2007) observed that under low boron concentrations, the arabidopsis boron exporter AtBOR1 facilitates boron transport from roots to shoots; while under high boron, AtBOR1 is degraded in the vacuole (Wakuta et al., 2015; Miwa et al., 2006, 2007). These findings indicate that boron concentration affects the dynamics of transporter proteins. A possible explanation is that boron itself interacts with the ribose in mRNA and changes its stability thereby affecting transcription and translation (Pommerrenig et al., 2015). On the other hand, Rámila et al. (2016) found that BoT1 transcripts increased under excess boron in Puccinellia. The BoT1 primers from that study (Rámila et al., 2016) were also used during validation of 


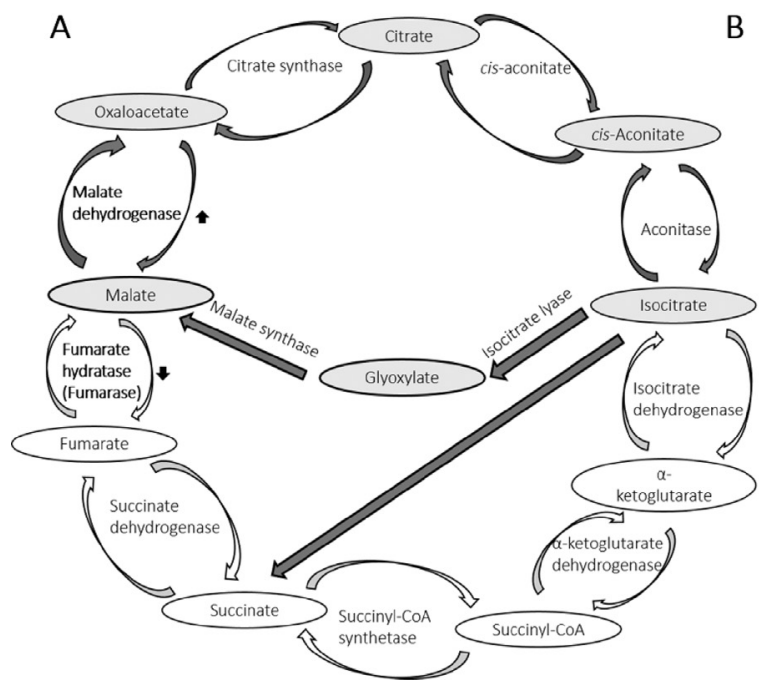

B

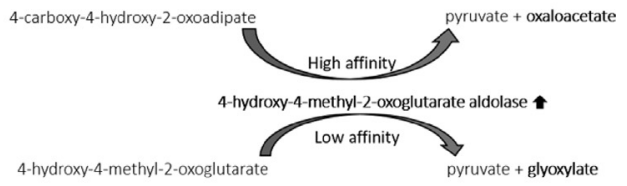

4-hydroxy-4-methyl-2-oxoglutarate pyruvate + glyoxylate

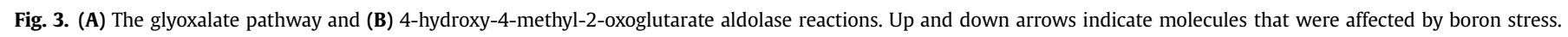

the bioinformatic analysis of the present study. Based on this analysis, no significant increase was observed with quantitative RTPCR (Fig. 2). This result was consistent with a previous study in barley (Sutton et al., 2007). Moreover, Tombuloglu et al. (2015) suggested that the Bot1 gene has seven duplicated paralogs and that their expression was not dependent on boron concentration in barley.

In our study, the $A$. thaliana BOR6 homolog (UniProtID: BOR6_ARATH), a transporter for boron efflux, was up-regulated in P. distans (Supplementary Table 6). AtBOR6 is a clade II transporter and contributes to tolerance by excluding boron under high boron conditions (Afzal et al., 2016). Since P. distans is a hyperaccumulator, our BOR6 homolog may also be working to exclude boron or it may not be a true homolog and have a different function. For example, it may facilitate boron transport from root to shoot or transport boron from the cytoplasm into the cell wall. Clade I boron transporters have a di-leucine motif which is necessary for polarity and borondependent vacuolar sorting of transporter protein (AtBOR1) (Wakuta et al., 2015). On the other hand, clade II motif similarities and their functions in boron transport have not yet been identified. Therefore, it would be interesting to obtain a full length sequence of the $P$. distans BOR6 homolog to compare with the other Clade II transporters to identify any possible conserved motifs and/or significant differences with the Puccinellia BOR6 and to elucidate their potential effects on boron hyperaccumulation/tolerance mechanisms.

Major intrinsic proteins (MIPs), also known as aquaporin proteins, facilitate the transport of water across biological membranes and are permeable to other small, uncharged molecules such as glycerol, solutes and ions (Pommerrenig et al., 2015; Babaoglu et al., 2004). MIPs are categorized into seven subgroups in the plant kingdom including NOD26-like intrinsic proteins (NIPs) and tonoplast intrinsic proteins (TIPs) transporters (Afzal et al., 2016; Pérez Di Giorgio et al., 2016). NIPs are generally located in the plasma membrane and endoplasmic reticulum; TIPs are localized in the vacuole membrane (tonoplast) and thylakoid inner membrane (Afzal et al., 2016). NIPs are the most divergent plant MIP subfamily with respect to their substrate specificities and amino acid sequences and are selectively permeable to metalloids (Pommerrenig et al., 2015). Specifically, NIP II and NIP III actively transport boron. In our study, two types of aquaporins were identified, one of them was a homolog of an 0 . sativa NIP (UniProtID: Q949A7_ORYSA; Transcript Name: TRINITY_DN210823_c0_g3_i1:4-269) and the other was a homolog of an A. thaliana TIP (UniProtID: TIP13_ARATH; Transcript Name: TRINITY_DN222891_c3_g1_i1:0-216) (Supplementary Table 6). Yildirim and Uylas (2016) found that NIP transcripts were downregulated under boron stress in poplar (Yildirim and Uylas, 2016), a result that was also seen in arabidopsis for AtTIP5; 1 (Pang et al., 2010). However, in our work, the O. sativa homolog NIP-type aquaporin was up-regulated in $P$. distans under boron stress. Similar up-regulation was observed in $P$. frigida (Rámila et al., 2016). Overexpression of aquaporins has been associated with tolerance to distinct stress conditions. For example, when PgTIP1 was overexpressed in A. thaliana, plants showed increased tolerance to salt and drought stress but decreased tolerance to cold (Peng et al., 2007). Therefore, the O. sativa NIP aquaporin could be responsible for cellular boron uptake in P. distans. Moreover, Kato et al. (2009) suggested that both NIP5; 1 and BOR1 enhancement are effective strategies to improve boron flow (Kato et al., 2009). Although the bidirectional activity of PIPs (plasma membrane intrinsic proteins) was demonstrated in rice under boron toxicity (Mosa et al., 2016; Fitzpatrick and Reid, 2009), none of these transcripts were identified in the present study.

Not only boron transporter proteins but also ABC-type transporter proteins were regulated in excess boron in this and previous studies (Padmanabhan et al., 2012; Rámila et al., 2016). ABC transporters have substrate specificities that vary from carbohydrates to ions and some are specialized to transport ions and solutes. For example, mntABCD is selectively permeable for manganese (Baumgart and Frunzke, 2015). In our study, five different $A$. thaliana $\mathrm{ABC}$ transporters (ABC-B, C, E, F, I) were differentially expressed under boron stress. All of the $A B C$ transporters were up-regulated with the exception of AB17I_ARATH. Rámila et al. (2016) suggested that ABC-type transporters are involved in detoxification and protection of plants from the harmful effect of boron stress (Rámila et al., 2016). The activation of this transporter group may also be due to the fact that $\mathrm{ABC}$ transporters are permeable to a wide variety of substrates and some of them may be able to transport boron. Moreover, they can transport boron-binding molecules such as sorbitol. Thus, ABC transporters may be a significant component of the boron hyperaccumulation mechanism in $P$. distans.

Under excess boron, a sulfate transporter was down-regulated in P. distans (UniProtID: Q8S317_ORYSA). Sulfate transport mechanisms are divided into four groups. The first is sulfate co-transport with a proton, the second is co-transport with sodium $(\mathrm{Na})$, the 
third is sulfate antiport with an anion and the fourth is ATPdependent $A B C$ transporter-mediated sulfate uptake (Takahashi et al., 2011). Sulfate transporters play important roles in response to metal stresses in a metal-specific manner (Kumar et al., 2011). Sulfate transporters are also responsible for transport of some oxyanions, such as molybdate and chromate (Appenroth et al., 2008; Fitzpatrick et al., 2008; Kumar et al., 2011). Kumar et al. (2011) observed that seven sulfate transporters were upregulated by arsenate and cadmium, ten were up-regulated by chromium and five by lead exposure. However, our study indicated down-regulation of a sulfate transporter in response to boron hyperaccumulation. Because excess boric acid will acidify the cellular environment, it is possible that less proton pumping is required to maintain cellular $\mathrm{pH}$.

\subsection{Hormone-related genes}

Plant hormones play critical roles by providing physiological and biochemical responses to stress factors (Colebrook et al., 2014). Abscisic acid (ABA) signaling is the central regulator of the abiotic stress resistance pathway in plants (Hubbard et al., 2010). Under drought conditions, plants cope with stress by increasing the amount of ABA causing stomatal closure and reduced transpiration (Cutler et al., 2010). In our dataset, A. thaliana homologs of ABArelated proteins such as phosphatase (UniProtID: P2C16_ARATH; Transcript Name: TRINITY_DN220107_c1_g6_i1:12-396) were down-regulated (Table S6). Reduction in ABA is associated with increases in cytosolic free calcium (Allen et al., 1999), calciumdependent proteins (Mustilli et al., 2002) and reactive oxygen species (Murata et al., 2001). According to our findings, calciumdependent proteins were up-regulated under excess boron, thus, supporting the relationship between $A B A$ and calcium-related proteins. In addition, it is well-known that both calcium and boron are important components of pectic polysaccharides in the cell wall (Matoh and Kobayashi, 1998). Therefore, an increase in boron could trigger up-regulation of calcium levels and calciumdependent proteins in order to maintain cell wall integrity. Ethylene and ABA are antagonists: ethylene triggers catabolism of ABA (Colebrook et al., 2014). In our analysis, five ethylene response proteins had increased expression under stress (Table S6). Thus as might be predicted, the ethylene response was up-regulated while ABA was down-regulated under boron stress.

Another plant hormone that has a role in stress defense is gibberellin (GA). GAs are involved in cell wall loosening in plants, thus contributing to cell expansion (Cosgrove, 1993; Park et al., 2015). In addition, GAs are inhibited under stress conditions to limit growth (Yamaguchi, 2008). For example, a reduced level of GA was observed under high salinity stress in A. thaliana (Magome et al., 2004). Interestingly, in our study, positive regulators of GAs (UniProtIDs: TSN1_ARATH, TSN2_ARATH; Transcript Names: TRINITY_DN151004, TRINITY_DN122991) had increased expression under boron stress (Table S6). The excess GAs produced under stress may maintain cell wall looseness as it is known that boron toxicity is usually accompanied by increased cell wall rigidity. Thus, GAs may have a critical role in $P$. distans boron tolerance by preventing cell rigidity, thereby allowing continued cell expansion and growth.

Other studies also identified signaling related proteins under excess boron (Padmanabhan et al., 2012; Rámila et al., 2016; Tombuloglu et al., 2015, 2017; Yildirim and Uylas, 2016). Additionally, calcium-related proteins were found to be increased in boron stress conditions (Tombuloglu et al., 2015). These results were consistent with the present study. Yildirim and Uylas (2016) suggested that calcium-related proteins could be involved with absorption and storage of excess boron in the cell.

\section{Conclusion}

P. distans is a non-model monocot that hyperaccumulates boron. Because knowledge of this species is limited, we used a transcriptomics approach to elucidate boron stress tolerance and potential hyperaccumulation mechanisms in $P$. distans. This analysis indicated that stress-related, cell wall, transporter and hormone genes are important components of the plant's response to and accumulation of boron. Strong evidence was obtained that the boron tolerance and hyperaccumulation mechanisms of $P$. distans involve alterations in the malate pathway, changes in cell wall components that allow sequestration of excess boron without toxic effects, and at least one putative boron transporter and two putative aquaporins. These results provide insights into the underlying boron stress responsive mechanism in P. distans shoots. Transgenic overexpression of the boron transporter and aquaporins in $P$. distans or determination of their subcellular localization will provide deeper information about their direct role in the boron hyperaccumulation mechanism. A greater understanding of hyperaccumulation/tolerance mechanism(s) should provide strategies for coping with boron stress toxicity in agriculturally important cereals. Thus, while $P$. distans itself might be used to eliminate excess boron from soil, the tolerance mechanism(s) could be transferred to crop species to confer tolerance and/or to develop a faster-growing, higher biomass phytoremediation system.

\section{Author contributions}

SEO performed laboratory experiments; $\mathrm{MG}$ and $\mathrm{CH}$ performed bioinformatics analysis; $\mathrm{MB}$ provided plant materials; SD, JA and $\mathrm{AF}$ designed the study; SEO, $\mathrm{CH}$, JA and AF drafted and revised the manuscript. All authors read the manuscript and agreed upon the final version of the manuscript.

\section{Competing financial interests}

The authors declare that they have no competing financial interests.

\section{Acknowledgments}

We thank Amy Frary for critical reading of the manuscript. This work was supported by a grant from The Scientific and Technological Research Council of Turkey (project no. 113Z930).

\section{Appendix A. Supplementary data}

Supplementary data related to this article can be found at doi:10.1016/j.chemosphere.2018.02.070.

\section{References}

Afzal, Z., Howton, T., Sun, Y., Mukhtar, M., 2016. The roles of aquaporins in plant stress responses. J. Dev. Biol. 4, 9.

Ali, H., Khan, E., Sajad, M.A., 2013. Phytoremediation of heavy metals-concepts and applications. Chemosphere 91, 869-881.

Allen, G.J., Kuchitsu, K., Chu, S.P., Murata, Y., Schroeder, J.I., 1999. Arabidopsis abi1-1 and abi2-1 phosphatase mutations reduce abscisic acid-induced cytoplasmic calcium rises in guard cells. Plant Cell 11, 1785-1798.

Appenroth, K.J., Luther, A., Jetschke, G., Gabrys, H., 2008. Modification of chromate toxicity by sulphate in duckweeds (Lemnaceae). Aquat. Toxicol. 89, 167-171.

Babaoǧlu, M., Gezgin, S., Topal, A., Sade, B., Dural, H., 2004. Gypsophila sphaerocephala Fenzl ex Tchihat.: a boron hyperaccumulator plant species that may phytoremediate soils with toxic B levels. Turk. J. Bot. 28, 273-278.

Bar, C., 2015. Elucidation of Boron Hyperaccumulation and Tolerance Mechanisms in Puccinellia Distans ( Jacq.) Parl. Using Proteomics Approach. master's thesis. Izmir Institute of Technology, Urla, Izmir, Turkey.

Bartels, D., Sunkar, R., 2005. Drought and salt tolerance in plants. Crit. Rev. Plant Sci. 24, 23-58. 
Baskin, T.I., 2005. Anisotropic expansion of the plant cell wall. Annu. Rev. Cell Dev. Biol. 21, 203-222.

Bassüner, R., Bäumlein, H., Huth, A., Jung, R., Wobus, U., Rapoport, T.A., Saalbach, G., Müntz, K., 1988. Abundant embryonic mRNA in field bean (Vicia faba L.) codes for a new class of seed proteins: cDNA cloning and characterization of the primary translation product. Plant Mol. Biol. 11, 321-334.

Baumgart, M., Frunzke, J., 2015. The manganese-responsive regulator MntR represses transcription of a predicted ZIP family metal ion transporter in Corynebacterium glutamicum. FEMS Microbiol. Lett. 362, 1-10.

Beevers, D.G., Lip, G.Y., O'Brien, E.T., 2014. ABC of Hypertension. John Wiley \& Sons, West Sussex, UK.

Binns, D., Dimmer, E., Huntley, R., Barrell, D., O'Donovan, C., Apweiler, R., 2009, QuickGO: a web-based tool for Gene Ontology searching. Bioinformatics 25, 3045-3046.

Blande, D., Halimaa, P., Tervahauta, A.I., Aarts, M.G., Kärenlampi, S.O., 2017. De novo transcriptome assemblies of four accessions of the metal hyperaccumulator plant Noccaea caerulescens. Scientific Data 4, 160131.

BOREN - Turkish National Boron Research Institute. 2012. Reserves - BOREN - National Boron Research Institute. URL http://www.boren.gov.tr/en/boron/ reserves. [accessed 1 January 2017].

Chen, Y., Zhi, J., Zhang, H., Li, J., Zhao, Q., Xu, J., 2017. Transcriptome analysis of Phytolacca americana L. in response to cadmium stress. PLoS One 12 (9), e0184681.

Colebrook, E.H., Thomas, S.G., Phillips, A.L., Hedden, P., 2014. The role of gibberellin signalling in plant responses to abiotic stress. J. Exp. Biol. 217, 67-75.

Conesa, A., Götz, S., García-Gómez, J.M., Terol, J., Talón, M., Robles, M., 2005. Blast2GO: a universal tool for annotation, visualization and analysis in functional genomics research. Bioinformatics 21, 3674-3676.

Cosgrove, D.J., 1993. Water uptake by growing cells: an assessment of the controlling roles of wall relaxation, solute uptake, and hydraulic conductance. Int. J. Plant Sci. 154, 10-21.

Cosgrove, D.J., 2005. Growth of the plant cell wall. Nat. Rev. Mol. Cell Biol. 6, $850-861$.

Cutler, S.R., Rodriguez, P.L., Finkelstein, R.R., Abrams, S.R., 2010. Abscisic acid: emergence of a core signaling network. Annu. Rev. Plant Biol. 61, 651-679.

Dannel, F., Pfeffer, H., Römheld, V., 2000. Characterization of root boron pools, boron uptake and boron translocation in sunflower using the stable isotopes $10 \mathrm{~B}$ and 11B. Aust. J. Plant Physiol. 27, 397-405.

Fitzpatrick, K.L., Reid, R.J., 2009. The involvement of aquaglyceroporins in transport of boron in barley roots. Plant Cell Environ. 32, 1357-1365.

Fitzpatrick, K.L., Tyerman, S.D., Kaiser, B.N., 2008. Molybdate transport through the plant sulfate transporter SHST1. FEBS Lett. 582, 1508-1513.

Goff, L., Trapnell, C., Kelley, D.R., 2013. cummeRbund: Analysis, Exploration, Manipulation, and Visualization of Cufflinks High-throughput Sequencing Data. R Package Version 2.16.0. https://bioconductor.org/packages/devel/bioc/ vignettes/cummeRbund/inst/doc/cummeRbund-manual.pdf. (Accessed 1 January 2017)

Goldbach, H.E., 1997. A critical review on current hypotheses concerning the role of boron in higher plants: suggestions for further research and methodological requirements. J. Trace Microprobe Tech. 15, 51-91.

Goldbach, H.E., Yu, Q., Wingender, R., Schulz, M., Wimmer, M., Findeklee, P., Baluska, F., 2001. Rapid response reactions of roots to boron deprivation. J. Soil Sci. Plant Nutr. 164, 173-181.

Haas, B.J., Papanicolaou, A., Yassour, M., Grabherr, M., Blood, P.D., Bowden, J., Couger, M.B., Eccles, D., Li, B., Lieber, M., MacManes, M.D., Ott, M., Orvis, J. Pochet, N., Strozzi, F., Weeks, N., Westerman, R., William, T., Dewey, C.N., Henschel, R., LeDuc, R.D., Friedman, N., Regev, A., 2013. De novo transcript sequence reconstruction from RNA-seq using the Trinity platform for reference generation and analysis. Nat. Protoc. 8, 1494-1512.

Hanaoka, H., Uraguchi, S., Takano, J., Tanaka, M., Fujiwara, T., 2014. OsNIP3;1, a rice boric acid channel, regulates boron distribution and is essential for growth under boron-deficient conditions. Plant J. 78, 890-902.

Hoagland, D.R., Arnon, D.I., 1950. The water-culture method for growing plants without soil. Calif. Agr. Exp. Sta. 347, 1-32.

Hubbard, K.E., Nishimura, N., Hitomi, K., Getzoff, E.D., Schroeder, J.I., 2010. Early abscisic acid signal transduction mechanisms: newly discovered components and newly emerging questions. Genes Dev. 24, 1695-1708.

Jamjod, S., 1996. Genetics of Boron Tolerance in Durum Wheat. doctor of philosophy thesis. The University of Adelaide, Adelaide, Australia.

Jenkin, M.J., 1993. Genetics of Boron Tolerance in Barley. doctor of philosophy thesis]. University of Adelaide, Adelaide, Australia].

Kanehisa, M., 2002. The KEGG database. In: Bock, G., Goode, J.A. (Eds.), In Silico Simulation of Biological Processes, vol. 247. John Wiley \& Sons, Chichester, UK. Novartis Foundation Symposium.

Kato, Y., Miwa, K., Takano, J., Wada, M., Fujiwara, T., 2009. Highly boron deficiencytolerant plants generated by enhanced expression of NIP5;1, a boric acid channel. Plant Cell Physiol. 50, 58-66.

Kumar, R.G., Shah, K., Dubey, R.S., 2000. Salinity induced behavioural changes in malate dehydrogenase and glutamate dehydrogenase activities in rice seedlings of differing salt tolerance. Plant Sci. 156, 23-34.

Kumar, S., Asif, M.H., Chakrabarty, D., Tripathi, R.D., Trivedi, P.K., 2011. Differential expression and alternative splicing of rice sulphate transporter family members regulate sulphur status during plant growth, development and stress conditions. Funct. Integr. Genom. 11, 259-273.

Langmead, B., Salzberg, S.L., 2012. Fast gapped-read alignment with Bowtie 2. Nat.
Meth. 9, 357-359.

Larkin, M.A., Blackshields, G., Brown, N.P., Chenna, R., Mcgettigan, P.A McWilliam, H., Valentin, F., Wallace, I.M., Wilm, A., Lopez, R., Thompson, J.D. Gibson, T.J., Higgins, D.G., 2007. Clustal W and clustal X version 2.0. Bioinformatics 23, 2947-2948.

Le, S.Q., Gascuel, O., 2008. An improved general amino acid replacement matrix Mol. Biol. Evol. 25, 1307-1320.

Levy, S., Staehelin, L.A., 1992. Synthesis, assembly and function of plant cell wall macromolecules. Curr. Opin. Cell Biol. 4, 856-862.

Loewus, F.A., Loewus, M.W., 1983. myo-Inositol: its biosynthesis and metabolism. Annu. Rev. Physiol. 34, 137-161.

Loewusa, F.A., Murthy, P.P.N., 2000. myo-Inositol metabolism in plants. Plant Sci. 150, 1-19.

Magome, H., Yamaguchi, S., Hanada, A., Kamiya, Y., Oda, K., 2004. Dwarf and delayed-flowering 1, a novel Arabidopsis mutant deficient in gibberellin biosynthesis because of overexpression of a putative AP2 transcription factor Plant J. 37, 720-729.

Marschner, H., Marschner, P., 2012. Marschner's Mineral Nutrition of Higher Plants third ed. Elsevier/Academic Press, London, UK.

Martin, M., 2011. Cutadapt removes adapter sequences from high-throughput sequencing reads. EMBnet. J. 17, 10-12.

Maruyama, K., 1990. Purification and properties of 4-hydroxy-4-methyl-2 oxoglutarate aldolase from Pseudomonas ochraceae grown on phthalate. J. Biochem. 108, 327-333.

Matoh, T., 2001. Boron in plant nutrition and cell wall development. In: Ae, N. Arihara, J., Okada, K., Srinivasan, A. (Eds.), Plant Nutrient Acquisition. Springer Tokyo, Japan, pp. 227-250.

Matoh, T., Kobayashi, M., 1998. Boron and calcium, essential inorganic constituents of pectic polysaccharides in higher plant cell walls. J. Plant Res. 111, 179-190.

Mi, H., Huang, X., Muruganujan, A., Tang, H., Mills, C., Kang, D., Thomas, P.D., 2017 PANTHER version 11: expanded annotation data from Gene Ontology and Reactome pathways, and data analysis tool enhancements. Nucleic Acids Res. 45, $1-15$.

Miwa, K., Takano, J., Fujiwara, T., 2006. Improvement of seed yields under boronlimiting conditions through overexpression of BOR1, a boron transporter for xylem loading, in Arabidopsis thaliana. Plant J. 46, 1084-1091.

Miwa, K., Takano, J., Omori, H., Seki, M., Shinozaki, K., Fujiwara, T., 2007. Plants tolerant of high boron levels. Science 318, 1417.

Mosa, K.A., Kumar, K., Chhikara, S., Musante, C., White, J.C., Dhankher, O.P., 2016. Enhanced boron tolerance in plants mediated by bidirectional transpor through plasma membrane intrinsic proteins. Sci. Rep-Uk 6, 21640.

Murata, Y., Pei, Z.M., Mori, I.C., Schroeder, J., 2001. Abscisic acid activation of plasma membrane $\mathrm{Ca}^{2+}$ channels in guard cells requires cytosolic $\mathrm{NAD}(\mathrm{P}) \mathrm{H}$ and is differentially disrupted upstream and downstream of reactive oxygen species production in abi1-1 and abi2-1 protein phosphatase 2C mutants. Plant Cell 13 , 2513-2523.

Mustilli, A.C., Merlot, S., Vavasseur, A., Fenzi, F., Giraudat, J., 2002. Arabidopsis OST1 protein kinase mediates the regulation of stomatal aperture by abscisic acid and acts upstream of reactive oxygen species production. Plant Cell 14, 3089-3099.

Nable, R.O., Bañuelos, G.S., Paull, J.G., 1997. Boron toxicity. Plant Soil 193, 181-198.

Neto, J.B.D.A., Hurtado-Perez, M.C., Wimmer, M.A., Frei, M., 2017. Genetic factors underlying boron toxicity tolerance in rice: genome-wide association study and transcriptomic analysis. J. Exp. Bot. 68, 687-700.

Ozturk, M., Sakcali, S., Gucel, S., Tombuloglu, H., 2010. Boron and plants. In: Ashraf, M., Ozturk, M., Ahmad, S.A. (Eds.), Plant Adaptation and Phytoremediation. Springer, Dordrecht, Netherland, pp. 275-311.

Padmanabhan, P., Babaoglu, M., Terry, N., 2012. A comparative transcriptomic analysis of the extremely boron tolerant plant Puccinellia distans with the moderately boron tolerant Gypsophila arrostil. Plant Cell Rep. 31, 1407-1413.

Padmavathiamma, P.K., Li, L.Y., 2007. Phytoremediation technology: hyperaccumulation metals in plants. Water Air Soil Pollut. 184, 105-126.

Pang, Y.Q., Li, L.J., Ren, F., Lu, P.L., Wei, P.C., Cai, J.H., Xin, L., Zhang, J., Chen, J., Wang, X., 2010. Overexpression of the tonoplast aquaporin AtTIP5;1 conferred tolerance to boron toxicity in arabidopsis. J. Genet. Genom. 37, 389-397.

Park, J., Cui, Y., Kang, B.H., 2015. AtPGL3 is an Arabidopsis BURP domain protein that is localized to the cell wall and promotes cell enlargement. Front. Plant Sci. 6 , 412.

Paull, J.G., Nable, R.O., Rathjen, A.J., 1992. Physiological and genetic control of the tolerance of wheat to high concentrations of boron and implications for plant breeding. Plant Soil 146, 251-260.

Peng, Y., LinWeiming, W., Arora, C., 2007. Overexpression of a Panax ginseng tonoplast aquaporin alters salt tolerance, drought tolerance and cold acclimation ability in transgenic Arabidopsis plants. Planta 226, 729-740.

Pérez Di Giorgio, J.A., Barberini, M.L., Amodeo, G., Muschietti, J.P., 2016. Pollen aquaporins: what are they there for? Plant Signal. Behav. 11 (9), e1217375.

Pommerrenig, B., Diehn, T.A., Bienert, G.P., 2015. Metalloido-porins: essentiality of nodulin 26-like intrinsic proteins in metalloid transport. Plant Sci. 238, $212-227$.

Rámila, C.D.P., Leiva, E.D., Bonilla, C.A., Pasténa, P.A., Pizarro, G.E., 2015. Boron accumulation in Puccinellia frigida, an extremely tolerant and promising species for boron phytoremediation. J. Geochem. Explor. 150, 25-34.

Rámila, C.D.P., Contreras, S.A., Di Domenico, C., Molina-Montenegro, M.A., Vega, A. Handford, M., Bonilla, C.A., Pizarro, G.E., 2016. Boron stress response and accumulation potential of the extremely tolerant species Puccinellia frigida. J. Hazard Mater. 317, 476-484. 
Reid, R., 2007. Identification of boron transporter genes likely to be responsible for tolerance to boron toxicity in wheat and barley. Plant Cell Physiol. 48, 1673-1678.

Ryan, J., Rashid, A., 2006. Application of soil and plant analysis for applied research and development in West Asia-North Africa: an international center's perspective. Commun. Soil Sci. Plant Anal. 37, 2185-2198.

Ryan, P., Delhaize, E., Randall, P., 1995. Characterisation of Al-stimulated efflux of malate from the apices of Al-tolerant wheat roots. Planta 196, 103-110.

Sang, W., Huang, Z.R., Qi, Y.P., Yang, L.T., Guo, P., Chen, L.S., 2015. An investigation of boron-toxicity in leaves of two citrus species differing in boron-tolerance using comparative proteomics. J. Proteomics 123, 128-146.

Schmidt, A.M., Sengupta, N., Saski, C.A., Noorai, R.E., Baldwin, W.S., 2017. RNA sequencing indicates that atrazine induces multiple detoxification genes in Daphnia magna and this is a potential source of its mixture interactions with other chemicals. Chemosphere 189, 699-708.

Seki, M., Umezawa, T., Urano, K., Shinozaki, K., 2007. Regulatory metabolic networks in drought stress responses. Curr. Opin. Plant Biol. 10, 296-302.

Shinozaki, K., Yamaguchi-Shinozaki, K., 2007. Gene networks involved in drought stress response and tolerance. J. Exp. Bot. 58, 221-227.

Shinozaki, K., Yamaguchi-Shinozaki, K., Seki, M., 2003. Regulatory network of gene expression in the drought and cold stress responses. Curr. Opin. Plant Biol. 6, 410-417.

Stiles, A.R., Bautista, D., Atalay, E., Babaoğlu, M., Terry, N., 2010. Mechanisms of boron tolerance and accumulation in plants: a physiological comparison of the extremely boron-tolerant plant species, Puccinellia distans, with the moderately boron-tolerant Gypsophila arrostil. Environ. Sci. Technol. 44, 7089-7095.

Sutton, T., Baumann, U., Hayes, J., Collins, N.C., Shi, B.J., Schnurbusch, T., Hay, A Mayo, G., Pallotta, M., Tester, M., Langridge, P., 2007. Boron-toxicity tolerance in barley arising from efflux transporter amplification. Science 318, 1446-1449.

Taji, T., Ohsumi, C., Iuchi, S., Seki, M., Kasuga, M., Kobayashi, M., YamaguchiShinozaki, K., Shinozaki, K., 2002. Important roles of drought- and coldinducible genes for galactinol synthase in stress tolerance in Arabidopsis thaliana. Plant J. 29, 417-426.

Takahashi, H., Buchner, P., Yoshimoto, N., Hawkesford, M.J., Shiu, S.H., 2011. Evolutionary relationships and functional diversity of plant sulfate transporters. Front. Plant Sci. 2, 119.

Takano, J., Yamagami, M., Noguchi, K., Hayashi, H., Fujiwara, T., 2001. Preferential translocation of boron to young leaves in Arabidopsis thaliana regulated by the BOR1 gene. Soil Sci. Plant Nutr. 47, 345-357.

Takano, J., Wada, M., Ludewig, U., Schaaf, G., von Wirén, N., Fujiwara, T., 2006. The Arabidopsis major intrinsic protein NIP5:1 is essential for efficient boron uptake and plant development under boron limitation. Plant Cell 18, 1498-1509.

Takano, J., Miwa, K., Fujiwara, T., 2008. Boron transport mechanisms: collaboration of channels and transporters. Trends Plant Sci. 13, 451-457.

Tamura, K., Stecher, G., Peterson, D., Filipski, A., Kumar, S., 2013. MEGA6: molecular evolutionary genetics analysis version 6.0. Mol. Biol. Evol. 30, 2725-2729.

Tanabe, M., Kanehisa, M., 2012. Using the KEGG database resource. Curr. Protoc. Bioinform. 1-12. https://doi.org/10.1002/0471250953.bi0112s38.

Tanaka, M., Fujiwara, T., 2008. Physiological roles and transport mechanisms of boron: perspectives from plants. Pflugers. Arch. 456, 671-677.

Tian, J.H., Xue, B., Hu, J.H., Li, J.X., Cheng, X.Y., Hu, J.S., Li, F.C., Chen, Y.H., Li, B., 2017. Exogenous substances regulate silkworm fat body protein synthesis through MAPK and PI3K/Akt signaling pathways. Chemosphere 171, 202-207.
Tombuloglu, G., Tombuloglu, H., Sakcali, M.S., Unver, T., 2015. High-throughput transcriptome analysis of barley (Hordeum vulgare) exposed to excessive boron. Gene 557, 71-81.

Tombuloglu, H., Tombuloglu, G., Sakcali, M.S., Turkan, A., Hakeem, K.R., Alharby, H.F., Fahd, S., Abdul, W.M., 2017. Proteomic analysis of naturally occurring boron tolerant plant Gypsophila sphaerocephala L. in response to high boron concentration. J. Plant Physiol. 216, 212-217.

Trapnell, C., Roberts, A., Goff, L., Pertea, G., Kim, D., Kelley, D.R., Pimentel, H., Salzberg, S.L., Rinn, S.L., Pachter, L., 2012. Differential gene and transcript expression analysis of RNA-seq experiments with TopHat and Cufflinks. Nat. Protoc. 7, 562-578.

Treacy, B.K., Hattori, J., Prud'homme, I., Barbour, E., Boutilier, K., Baszczynski, C.L., Huang, B., Johnson, D.A., Miki, B.L., 1997. Bnm1, a Brassica pollen-specific gene. Plant Mol. Biol. 34, 603-611.

Unver, T., Bozkurt, O., Akkaya, M.S., 2008. Identification of differentially expressed transcripts from leaves of the boron tolerant plant Gypsophila perfoliata L. Plant Cell Rep. 27, 1411-1422.

Uraguchi, S., Fujiwara, T., 2011. Significant contribution of boron stored in seeds to initial growth of rice seedlings. Plant Soil 340, 435-442.

Wakuta, S., Mineta, K. Amano, T., Toyoda, A., Fujiwara, T., Naito, S., Takano, J., 2015 Evolutionary divergence of plant borate exporters and critical amino acid residues for the polar localization and boron-dependent vacuolar sorting of AtBOR1. Plant Cell Physiol. 56, 852-862.

Wang, Y., Yang, C., Liu, G., Jiang, J., 2007. Development of a cDNA microarray to identify gene expression of Puccinellia tenuiflora under saline-alkali stress. Plant Physiol. Biochem. 45, 567-576.

Wang, H., Zhou, L., Fu, Y. Cheung, M., Wong, F., Phang, T., Sun, Z., Lam, H.M., 2012 Expression of an apoplast-localized BURP-domain protein from soybean (GmRD22) enhances tolerance towards abiotic stress. Plant Cell Environ. 35, 1932-1947.

Xu, H., Li, Y., Yan, Y., Wang, K., Gao, Y., Hu, Y., 2010. Genome-scale identification of soybean BURP domain-containing genes and their expression under stress treatments. BMC Plant Biol. 10, 1.

Yamaguchi, S., 2008. Gibberellin metabolism and its regulation. Annu. Rev. Plant Biol. 59, 225-251.

Yamaguchi-Shinozaki, K., Shinozaki, K., 1993. The plant hormone abscisic acid mediates the drought-induced expression but not the seed-specific expression of $r d 22$, a gene responsive to dehydration stress in Arabidopsis thaliana. Mol. Gen. Genet. 238, 17-25.

Yang, Q., Shohag, M.J.I., Feng, Y., He, Z., Yang, X., 2017. Transcriptome comparison reveals the adaptive evolution of two contrasting ecotypes of $\mathrm{Zn} / \mathrm{Cd}$ hyperaccumulator Sedum alfredii hance. Front. Plant Sci. 8.

Yau, S.K., Nachit, M.M., Ryan, J., Hamblin, J., 1995. Phenotypic variation in borontoxicity tolerance at seedling stage in durum wheat (Triticum durum). Euphytica 83, 185-191.

Yildirim, K., Uylas, S., 2016. Genome-wide transcriptome profiling of black poplar (Populus nigra L.) under boron toxicity revealed candidate genes responsible in boron uptake, transport and detoxification. Plant Physiol. Biochem. 109, $146-155$.

Zheng, L., 1992. The $\beta$ subunit of tomato fruit polygalacturonase isoenzyme 1: isolation, characterization, and identification of unique structural features. Plant Cell 4,1147-1156. 